\title{
Achados clínicos e radiográficos de duas anomalias dentárias de forma em um paciente: relato de caso
}

\author{
Clinical and radiographic findings of two dental anomalies of shape in a patient: case report \\ Hallazgos clínicos y radiográficos de dos anomalías dentales de forma en un paciente: reporte de
}

\author{
Amanda Marinho Chaves Costa \\ ORCID: https://orcid.org/0000-0002-6566-4890 \\ Centro Universitário Cesmac, Brasil \\ E-mail: amandamccosta@outlook.com \\ Denise Barboza de Souza \\ ORCID: https://orcid.org/0000-0003-2286-3002 \\ Centro Universitário Cesmac, Brasil \\ E-mail: edsonphilippebb@hotmail.com \\ Catarina Rodrigues Rosa de Oliveira \\ ORCID: https://orcid.org/0000-0001-9178-8902 \\ Centro Universitário Cesmac, Brasil \\ E-mail: catarinarosaodonto@hotmail.com \\ Áurea Valéria de Melo Franco \\ ORCID: https://orcid.org/0000-0002-5966-8617 \\ Centro Universitário Cesmac, Brasil \\ E-mail: aurea.franco@cesmac.edu.br \\ Enzo Lima Mella \\ ORCID: https://orcid.org/0000-0002-6527-7399 \\ Centro Universitário Cesmac, Brasil \\ E-mail: enzo.mella@hotmail.com \\ Isabela Alencar Delgado \\ ORCID: https://orcid.org/0000-0002-4987-226X \\ Centro Universitário Cesmac, Brasil \\ E-mail: Isabela.alencar2009@ hotmail.com \\ Maria Eduarda Silva Bezerra \\ ORCID: https://orcid.org/0000-0001-5165-4248 \\ Centro Universitário Cesmac, Brasil \\ E-mail:contatomariaeduarda@ outlook.com \\ Wanderson Thalles de Souza Braga \\ ORCID: https://orcid.org/0000-0003-3617-3950 \\ Centro Universitário Tiradentes, Brasil \\ E-mail: wanderson.thalles@souunit.com.br
}

\begin{abstract}
Resumo
As anomalias dentárias, definidas como alterações resultantes de diversos fatores etiológicos que atuam durante o desenvolvimento dentário ou adquiridas durante a vida. A geminação é uma anomalia dentária que descreve um dente de tamanho aumentado ou de formato incomum que parece ser composto por dois dentes. $\mathrm{O}$ taurodontismo se trata de uma anomalia de desenvolvimento que se apresenta como câmara pulpar alongada e canais radiculares curtos. Paciente M. J. C., 61 anos, leucoderma, compareceu ao consultório odontológico para avaliação de higiene oral. Ao exame intraoral foi possível observar clinicamente no dente 33 um aspecto semelhante a geminação. Foi solicitado radiografia periapical a fim de complementar o diagnóstico. Durante avaliação radiográfica foi possível observar na região do dente 46 uma diminuição dos canais radiculares e câmara pulpar aumentada, sinal patognomônico de taurodontismo. Não houve necessidade de intervenção terapêutica em relação às anomalias encontradas. O conhecimento do cirurgiãodentista acerca das características clínicas e radiográficas das anomalias dentárias são de fundamental importância para detecção de tais, bem como a condução terapêutica mais adequada para cada caso.
\end{abstract}

Palavras-chave: Anomalias maxilomandibulares; Anormalidades congênitas; Anormalidades dentárias.

\begin{abstract}
Dental anomalies, defined as alterations resulting from various etiological factors that act during dental development or acquired during life. Gemination is a dental anomaly that describes an enlarged or unusually shaped tooth that appears to consist of two teeth. Taurodontism is a developmental anomaly that presents as an elongated pulp chamber and short
\end{abstract}


root canals. Patient M.J.C., 61 years old, leucoderma, attended the dental office for oral hygiene assessment. On intraoral examination, it was possible to clinically observe an aspect similar to gemination in tooth 33 . Periapical radiography was requested to complement the diagnosis. During radiographic evaluation, it was possible to observe in the region of tooth 46 a decrease in the root canals and an increased pulp chamber, a pathognomonic sign of taurodontism. There was no need for therapeutic intervention regarding the anomalies found. Dentists' knowledge about the clinical and radiographic characteristics of dental anomalies is of fundamental importance for the detection of such anomalies, as well as the most appropriate therapeutic approach for each case.

Keywords: Maxillomandibular abnormalities; Congenital abnormalities; Tooth abnormalities.

\section{Resumen}

Anomalías dentales, definidas como alteraciones resultantes de diversos factores etiológicos que actúan durante el desarrollo dentario o adquiridas durante la vida. La geminación es una anomalía dental que describe un diente agrandado o de forma inusual que parece consistir en dos dientes. El taurodontismo es una anomalía del desarrollo que se presenta como una cámara pulpar alargada y conductos radiculares cortos. Paciente M.J.C., 61 años, leucoderma, acudió al consultorio odontológico para valoración de higiene bucal. En el examen intraoral se pudo observar clínicamente un aspecto similar a la geminación en el diente 33. Se solicitó radiografía periapical para complementar el diagnóstico. Durante la evaluación radiográfica se pudo observar en la región del diente 46 una disminución de los conductos radiculares y un aumento de la cámara pulpar, signo patognomónico de taurodontismo. No hubo necesidad de intervención terapéutica con respecto a las anomalías encontradas. El conocimiento de los odontólogos sobre las características clínicas y radiográficas de las anomalías dentarias es de fundamental importancia para la detección de dichas anomalías, así como el abordaje terapéutico más adecuado para cada caso.

Palabras clave: Anomalías maxilomandibulares; Anomalías congénitas; Anomalías dentarias.

\section{Introdução}

Ao avaliar a cavidade oral, o cirurgião-dentista, no momento em que se depara com aspectos anatômicos dentais que fujam da normalidade, deve saber conduzir o paciente ao tratamento adequado. Em conjunto com a anamnese e exame clínico, os exames complementares de imagem desempenham papel importante no diagnóstico de anomalias dentais. (Seabra, Macho, Pinto \& Soares, 2008).

As anomalias dentárias são conceituadas como irregularidades odontogênicas ou alteração dentárias (Garib, Filho, \& Lara, 2013) provenientes de problemas durante o processo de formação dentária (Uslu, Akcam, Evirgen, \& Cebeci, 2009), classificadas em: hereditárias, congênitas ou adquiridas (Bilge, Yesiltepe, Ağırman, Çağlayan \& Bilge, 2018). As hereditárias são alterações que ocorrem na fase de diferenciação celular, as alterações que acontecem na fase intrauterina são chamadas de congênitas e as adquiridas quando essas modificações acontecem no desenvolvimento após o nascimento (Andrade, Lima, Silva, Vasconcelos \& Vasconcelos, 2017) como é o caso do taurodontismo e geminação (Occhiena, 2015).

A geminação é a divisão de um único germe dentário na tentativa de gerar dois dentes, na separação parcial com aparência de duas coroas que partilham o mesmo canal (Regezi, Sciubba, \& Jordan, 2008). Dentes que apresentam esta anomalia, retratam aumento de tamanho, coroa dupla, bífida ou chanfrada, raiz única e canal radicular único (Silva, 2013; Moura, Negri, Simão, Dantas, \& Crepaldi, 2013; Carvalho, Silva, Duarte, \& Júnior, 2014; Neville, Damm, Allen, \& Bouquot, 2016). Esta alteração pode favorecer o surgimento de lesões cariosas, problemas periodontais, oclusais e estéticos, cabendo o tratamento específico para cada caso (Costa, 2015). Deve-se analisar os exames radiográficos minuciosamente, com atenção nas estruturas no canal radicular, amplitude da polpa para evitar complicações no tratamento e conservar o dente (Saxena, Pandey, \& Kamboj, 1998). Na ocorrência de geminação, a lâmina dental permanece entre os germes (Seabra, 2008; Carvalho et al., 2014; Costa, 2015).

A taurodontia representa uma ampliação do corpo e da câmara pulpar de um dente com mais de uma raiz, deslocamento apical do assoalho pulpar e da bifurcação das raízes (Neville, Damm, Allen \& Bouquot, 2016; Nair, Jayapraksh \& Patil, 2019). Se trata de uma variação na forma dos dentes, os quais se apresentam como coroas alongadas, embora Nair, Jayapraksh \& Patil (2019) relatem que clinicamente o dente aparenta estar normal. Pode ser encontrada isoladamente, em famílias, e em associação com síndromes tais como a síndrome de Down e a de Klinefelter (Regezi, Sciuba \& Jordan, 2008). 
Na radiografia periapical, essa alteração também pode ser classificada pela posição vertical do assoalho pulpar, como no hipotaurodontismo, que apresenta câmara pulpar aumentada sem afetar as raízes. No mesotaurodontismo, há um incremento maior da câmara pulpar com raízes mais curtas, mas elas permanecem separadas. O hipertaurodontismo é a forma que apresenta bifurcação ou trifurcação do terço apical da raiz que dificulta a localização e o acesso aos canais (Shaw, 1928; Bharti, Chandra, Tikku, \& Wadhwani, 2009).

Diante do exposto, o objetivo deste estudo é relatar um caso clínico de achado de anomalias dentárias de desenvolvimento através de um exame clínico e radiográfico, bem como a condução terapêutica do caso.

\section{Metodologia}

O presente estudo dispõe da apresentação clínica e radiográfica de taurodontismo e geminação por meio de relato de caso clínico (estudo de caso) com objetivos descritivos e uma abordagem qualitativa (Pereira et al., 2018). Com base nos dados de um prontuário da Liga Acadêmica de Diagnóstico por Imagem Odontológica do Centro Universitário Cesmac. Assim, em concordância com o conhecimento da prática de radiologia e com base na literatura científica sobre o tema abordado, considerando uma sequência de condução de diagnóstico do caso apresentado, propõe-se relatar um caso clínico de taurodontismo e geminação, com diagnóstico fundamentado por meio de exame clínicos, em associação com os exames complementares. O estudo teve o Termo de Consentimento Livre e Esclarecido - TCLE e foi aprovado pelo Comitê de Ética e Pesquisa com o parecer de número 4.790.104.

\section{Relato de caso}

Paciente M. J. C., leucoderma, sexo feminino, 61 anos de idade, compareceu ao consultório odontológico com a queixa de "Quero fazer avaliação na boca". Na anamnese a paciente não relatou sintomatologia dolorosa, mas relatou ser tabagista há pelo menos 35 anos e que fazia uso de prótese parcial removível na arcada superior. Ao exame intraoral foi possível observar aspecto de bifurcação do dente 33 (Figura 1).

Imagem 1: Fotografia frontal da face da paciente.

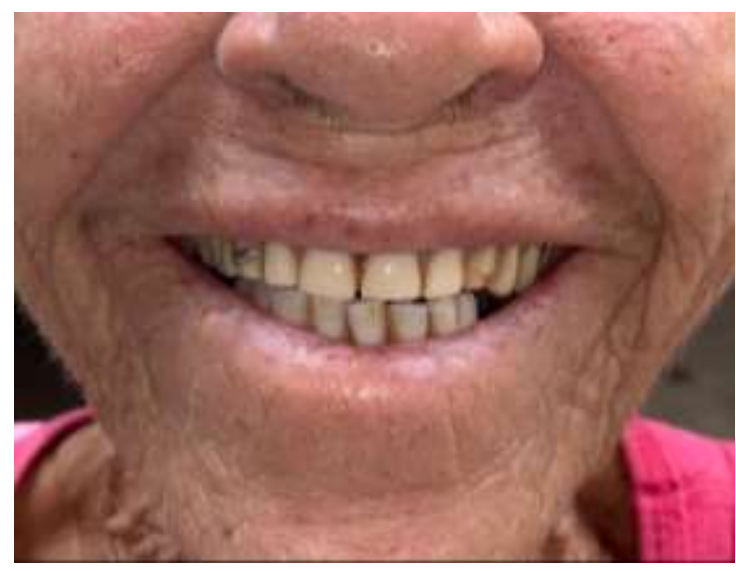

Fonte: Dados da pesquisa.

Ao exame clínico intra oral, foi observado que a paciente fazia uso de prótese parcial removível no arco superior, e foram observadas diversas lesões cariosas e a presença de anomalia dentária sugestiva de geminação no dente 33. A fim de se realizar uma avaliação radiográfica da cavidade bucal, foram solicitadas exames complementares de radiografias periapicais dos dentes $31,32,33,34,41,42,43$ e 46. Mediante a radiografia periapical da região do dente 33, observou-se uma tentativa de 
separação das coroas, ambas recebendo suporte de um único canal, característica marcante e decisiva da anomalia dentária de geminação (Imagem 2) e ampliação da câmara pulpar do dente 46, característica marcante de taurodontia (Imagem 3).

Imagem 2: Radiografia periapical da região do dente 33, que confirma o diagnóstico de taurodontismo no elemento

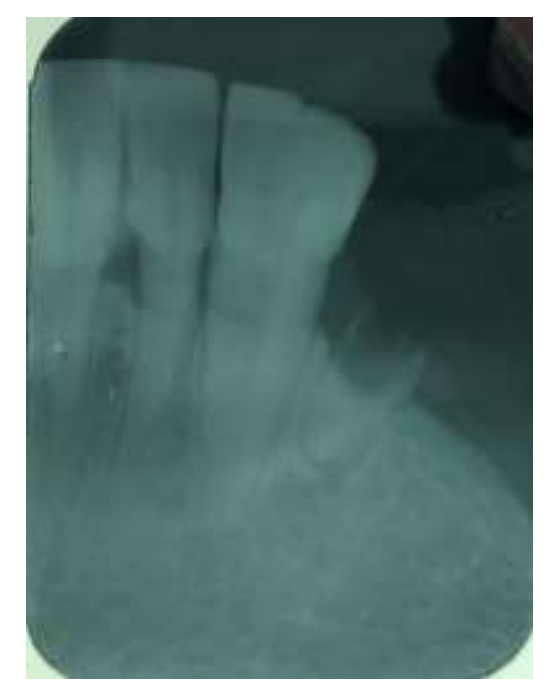

Fonte: Dados da pesquisa.

Imagem 3: Radiografia periapical do elemento 46, que confirma o diagnóstico de taurodontismo no elemento.

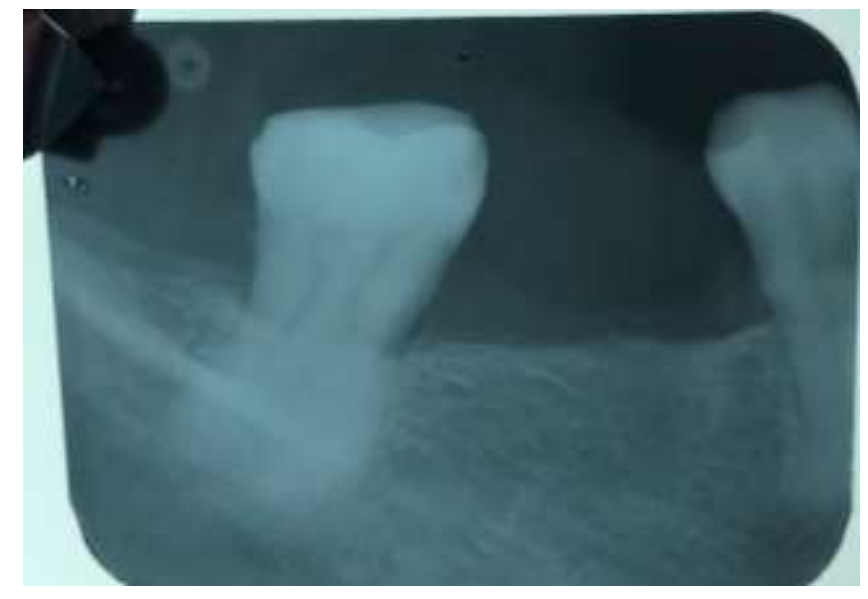

Fonte: Dados da pesquisa.

Não houve necessidade de intervenção terapêutica em relação às anomalias encontradas, cabendo apenas a orientação à paciente quanto ao cuidado na higienização do dente que apresentava geminação.

\section{Discussão}

As anomalias de desenvolvimento são classificadas em número, forma, tamanho e estrutura. Dentre as anomalias de desenvolvimento de forma tem-se como exemplo a taurodontia e a geminação (Occhiena, 2015).

A geminação é uma anomalia que ocorre com maior frequência em dentes decíduos, similar entre os sexos feminino e masculino e quando acomete a dentição permanente. Geralmente surge nos incisivos e caninos inferiores unilateralmente (Ruschel et al., 2011; Carvalho et al., 2014; Costa, 2015). E caracterizada pela tentativa de divisão de um único dente, e na radiografia é encontrado apenas um canal radicular. Diferentes formas de tratamento podem ser aplicadas, dependendo das 
características clínicas encontradas. (Regezi, Sciuba \& Jordan, 2008; Neville, Damm, Allen, \& Bouquot, 2009; Ruschel el at., 2011). Tais particularidades foram observadas neste relato de caso, o dente 33 apresentou características correspondentes à geminação.

$\mathrm{Na}$ literatura, não há concordância a respeito do tratamento mais viável para a geminação, o que se preconiza é a realização de um tratamento individualizado, onde deve ser considerado a anatomia individual de cada paciente. Os autores disponibilizam vários tratamentos, como restaurações, aplicação de selantes nos sulcos medianos, terapia pulpar, cortes e secções objetivando a diminuição do tamanho da coroa, tratamento ortodôntico, exodontias e próteses (Brook \& Winter, 1970; Ruschel, Bervian, Ferreira, \& Kramer, 2011). Ruschel et al (2011), Costa (2015), Neville, Damm, Allen, e Bouquot (2009) havendo relatos sobre a possível presença ocorrência de cárie no sulco da coroa bífida e o tratamento seria o selamento dos sulcos e das fissuras ou uma restauração. No entanto no caso descrito o dente 33 se apresentou hígido, não necessitando de tratamento.

A taurodontia, classificada como anomalia de forma, identificada por uma ampliação do corpo e um aumento oclusoapical da câmara pulpar do dente com mais de uma raiz conforme relato de diversos autores (Regezi, Sciuba \& Jordan, 2008; Neville et al., 2016; Nair, Jayapraksh \& Patil, 2019). Bharti, Chandra, Tikku e Wadhwani (2009), Neville, Damm, Allen, e Bouquot (2009), e Costa (2015). Os dentes mais frequentes acometidos são os molares permanentes. Esta anomalia está relacionada com algumas síndromes, como a síndrome de Down, Síndrome de Klinefelter, Síndrome de Mohr, Síndrome de Lowe, entre outras. Neville Neville, Damm, Allen, e Bouquot (2009) também afirmam que a taurodontia pode ocorrer de forma unilateral ou bilateral, não havendo predileção por gênero, havendo incidência frequente de casos de taurodontia em pacientes com hipodontia, fenda labial e fenda palatina. Neste referido caso, o dente afetado trata-se de um molar inferior (46) e se apresentou unilateralmente, dentro do padrão da literatura. No entanto, neste relato não se trata de um paciente com síndrome nem com hipodontia, fenda labial ou fenda palatina, casos relacionados com a anomalia. Esta, por sua vez, foi notada através de um achado radiográfico a partir da obtenção de radiografia periapical.

De acordo com Costa (2015) é importante reforçar a intervenção e terapêutica pelo cirurgião-dentista frente ao taurodontismo para que haja uma boa mastigação, oclusão satisfatória e estética ao paciente. Neville (2009), afirma que pacientes com esta anomalia, não necessitam de tratamento específico. No presente caso não houve intervenção no dente apresentado.

\section{Conclusão}

Os dados obtidos durante este estudo mostraram a importância de um diagnóstico clínico adequado, visando a melhoria da qualidade de vida do paciente. O taurodontismo tem relevância clínica em diversas áreas na odontologia como a endodontia que clinicamente, a anatomia variada e difícil do sistema de câmara e canal de um dente com taurodontismo complica seu manejo endodôntico. Desta forma, antes do tratamento, torna-se essencial fazer um diagnóstico preciso desta anomalia, por meio de um exame radiográfico cuidadoso. A morfologia anormal dos dentes geminados exige tratamento profilático e preventivo. Se faz necessário um exame minucioso por métodos clínicos e radiográficos que forneçam um diagnóstico preciso, dispondo o máximo de informações necessárias para estabelecimento do diagnóstico diferencial preciso, sendo o melhor tratamento com envolvimento de uma equipe multidisciplinar.

\section{Referências}

Andrade, C. E. d. S., Lima, I. H. L., Silva, I. V. d. S., Vasconcelos, M. G., \& Vasconcelos, R. G. (2017). As principais alterações dentárias de desenvolvimento (2nd ed.). Revista Salusvita, 36(2), 533-363.

Berbert, A. L., \& Mantese, S. A. (2005). Lúpus eritematoso cutâneo: aspectos clínicos e laboratoriais (3rd ed.). An Bras Dermatol, 80(2).

Bharti, R., Chandra, A., Tikku, A. P., \& Wadhwani, K. K. (2009). Taurodontism an endodontic challenge: a case report (2nd ed.). J Oral Sci. 51(3), 471-474. 
Bilge, N. H., Yeşiltepe, S., Ağırman, K. T., Çağlayan, F., Bilge, O. M. (2018). Investigation of prevalence of dental anomalies by using digital panoramic radiographs. Folia Morphol, 77(2):323-328.

Brook, A. H., \& Winter, G. B. (1970). Double teeth. A retrospective study of 'geminated' and 'fused' teeth in children. Br Dent J. 129(3), 123-30.

Costa, L. M. B. (2015). Avaliar a prevalência de anomalias dentárias congénitas (de desenvolvimento) na Clínica Universitária Egas Moniz (Dissertação de mestrado). Instituto Superior de Ciências da Saúde Egas Moniz, Monte de Caparica, Portugal.

Da Silva, L. O. G., Peixoto, L. A. d. O., Saldanha, M. J. d. A., \& Zerbinati, L. P. S. (2013). Supranumerários fusionados: relato de caso. Revista Bahiana de Odontologia, 4(1), 76-82.

De Carvalho, P. H. M., da Silva, B. C. d. B., Duarte, B. G., \& Júnior, H. V. d. R., (2014). Alterações de desenvolvimento dentário em relação à forma: relato de casos. Ciência Atual-Revista Científica Multidisciplinar das Faculdades São José, 3(1), 03-10.

Garib, D. G., Filho, O. G. d. S., \& Lara, T. S. (2013). Ortodontia interceptativa: protocolo de tratamento em duas fases. São Paulo, Artes Médicas.

Júnior, A. A. U., Cantisano, M. H., Klumb, E. M., Dias, E. P., \& da Silva, A. A. (2010). Achados bucais e laboratoriais em pacientes com lúpus eritematoso sistêmico: Oral and laboratorial findings in patients with systemic lupus erythematosus. J Bras Patol Med Lab, 46(6), 479-486.

Moura, L., Negri, M., Simão, T. M., Dantas, W. C. F., Crepaldi, A., (2013). Variações anatômicas que podem dificultar o tratamento endodôntico. Revista Faipe, $3(1), 61-68$.

Neville, B. W., Damm, D. D., Allen, C. M., \& Bouquot, J. E. (2009). Patologia oral \& maxilofacial (3nd ed.). Guanabara Koogan.

Neville, B. W., Damm, D. D., Allen, C. M., \& Bouquot, J. E. (2016). Patologia oral \& maxilofacial. Elsevier.

Nair R., Khasnis S. \& Patil J. D. (2019). Bilateral taurodontism in permanent maxillary first molar. Indian J Dent Res 30:314-7.

Occhiena, C. M. (2015). Anomalias Dentárias em Pacientes com Síndrome de Down. (Trabalho de Conclusão de Curso). Faculdade de Odontologia de Araçatuba, São Paulo, Brasil.

Regezi, J. A., Sciubba, \& Jordan. (2008). Patologia Bucal: Correlações clínico patológicas (3rd ed.). Guanabara koogan.

Ruschel, H. C., Bervian, J. Ferreira, S. H., \& Kramer, P. F. (2011). Dente decíduo duplo: relato de um caso atípico. RFO UPF, 16(1), 85-89.

Saxena, A., Pandey, R. K., \& Kamboj, M. (2008). Bilateral fusion of permanent mandibular incisors: a case report. J Indian Soc Pedod Prev Dent, 26(1), $32-33$. Seabra, M., Macho, V., Pinto, A., \& Soares, D. (2008). A importância das dentárias de desenvolvimento. Acta Pediatr Port, 39(5):195-200. http://dx.doi.org/10.25754/pjp.2008.4601.

Shaw, J. C. (1928). Taurodont teeth in South African Races. J Anat, 62(4), 476-498.

Uslu, O., Akcam, M. O., Evirgen, S., \& Cebeci, I. (2009). Prevalence of dental anomalies in various malocclusions. American Journal Orthodontics and Dentofacial Orthopaedics, 135(3), 328-335. 